\title{
Small hydrophobic protein of respiratory syncytial virus as a novel vaccine antigen
}

\author{
"Possibly the most intriguing features of human respiratory syncytial \\ virus are its ability to infect young infants in the presence of maternal \\ neutralizing antibodies and its ability to reinfect throughout life."
}

Keywords: human respiratory syncytial virus $\bullet$ small hydrophobic protein $\bullet$ vaccine

Human respiratory syncytial virus (HRSV) is the leading cause of lower respiratory tract infections and associated hospitalizations of infants worldwide [1]. HRSV infects the epithelium of the lung and actively initiates the shedding of infected cells without inducing major cytopathology [2]. When these infected cells are not efficiently cleared by macrophages or $\mathrm{T}$ cells, they form clumps that can obstruct the very narrow airways of young infants [3]. Possibly the most intriguing features of HRSV are its ability to infect young infants in the presence of maternal neutralizing antibodies and its ability to reinfect throughout life $[4,5]$. As such HRSV is also associated with excess morbidity and mortality in elderly people [6].

Why is it that the natural immune responses poorly protect against reinfection with HRSV? First, HRSV evades innate immune responses [7]. In addition, most HRSV infections are restricted to the upper respiratory tract, a site that is poorly accessed by circulating, potentially virus-neutralizing $\mathrm{IgG}$. There is some evidence to suggest that HRSV-neutralizing antibodies inversely correlate with severity of HRSV disease [8-10]. In line with this, prophylactic use of a monoclonal HRSV-neutralizing antibody reduces HRSV-associated hospitalizations of high-risk infants by approximately 50\% [11]. However, epidemiological and controlled HRSV challenge studies showed that infants and even healthy adults with high levels of serum antibodies directed against the neutralizing HRSV antigens $F$ and $G$, remained susceptible to HRSV infection $[8,9]$.

With these observations and the fact that very young infants are an important target group, it is not surprising that decades of HRSV research has not yet resulted in an effective vaccine. As HRSV neutralizing antibodies can provide a degree of protection, most subunit vaccine developments aim at inducing such antibodies. HRSV F and $G$ are the major surface proteins and are known to display neutralizing epitopes [12]. Recently, important progress has been made in developing RSV F and $G$ subunit vaccines, including the breakthrough of the production of recombinant HRSV $F$ protein that is stabilized in its prefusion conformation [13]. Compared to postfusion $\mathrm{F}$, immunization with prefusion $F$ induces higher HRSV-neutralizing antibodies in mice and in nonhuman primates. In addition, antibodies directed against the conserved cysteine noose of the HRSV G proteins, that contain a $\mathrm{CX} 3 \mathrm{C}$ motif, were shown to inhibit protein G-CX3CR interaction and reduce HRSV replication and disease [14]. Taking into account the partial immunity evoked by recurrent natural infections, it remains to be seen whether HRSV subunit vaccines solely based on the $\mathrm{F}$ and/or $\mathrm{G}$ proteins will be able to provide strong long-living immunity.

\section{Bert Schepens}

Department of Medical Protein Research, VIB, 9052, Ghent, Belgium and

Department of Biomedical Molecular Biology, Ghent University, 9052 Ghent, Belgium

\section{Michael Schotsaert}

Icahn School of Medicine at Mount Sinai, 1428 Madison Avenue, New York, NY 10029, USA

\section{Xavier Saelens}

Author for correspondence:

Department of Medical Protein

Research, VIB, 9052, Ghent, Belgium and

Department of Biomedical Molecular Biology, Ghent University, 9052 Ghent, Belgium

xavier.saelens@dmbr.vib-ugent.be 
Our studies have explored an alternative antibodyaccessible HRSV antigen; the ectodomain of the small hydrophobic protein $(\mathrm{SH})$. This membrane protein comprises 64 (HRSV subgroup A) or 65 (HRSV subgroup B) amino acids (AA) and is expressed by all members of the Pneumovirinae subfamiliy of the Paramyxoviridae family, including Bovine RSV, Ovine RSV and Human Metapneumovirus. In infected cells $\mathrm{SH}$ accumulates at the golgi, endoplasmic reticulum and plasma membrane but is hard to detect in HRSV virions [15]. A minor fraction of $\mathrm{SH}$ proteins is modified by glycosylation or expressed as a truncated variant [16]. SH forms pentameric cation-selective ion channels and has been reported to activate the NLPR3 inflammasome and to inhibit apoptosis and TNF signaling [17-19]. Evidence suggests that the protein is dispensable for in vitro replication of HRSV but HRSV lacking $\mathrm{SH}$ are attenuated in vivo [20].

The short (HRSV A: 23 AA; HRSV B: 24 AA) SH ectodomain ( $\mathrm{SHe}$ ) appears to be weakly immunogenic upon natural infections. Following HRSV infection, only about half of the children developed a rise in SHespecific serum IgG [21]. Likewise, SHe-specific IgG levels are very low in convalescent sera from HRSV infected $\mathrm{BALB} / \mathrm{c}$ mice, cotton rats and in human reference sera [22]. Therefore, we made SHe immunogenic by conjugating synthetic SHe peptide to keyhole limpet hemocyanin. Immunization of $\mathrm{BALB} / \mathrm{c}$ mice and cotton rats with this conjugate induced high levels of SHe-specific IgG and this immune response was associated with reduced pulmonary HRSV replication and protection against morbidity. However, SHe immune sera, which confers protection against HRSV challenge to naive mice, lack HRSV-neutralizing activity in cell line cultures. We speculate that the low abundance of $\mathrm{SH}$ on virions and potential shielding by $\mathrm{F}$ and $\mathrm{G}$ contribute to this lack of neutralization $[15,22]$. Furthermore, SH-deficient viruses replicate very efficiently in vitro [23]. It is possible that SHe-specific IgG would interfere with HRSV replication in primary human bronchial epithelial cells differentiated on an air-liquid interface. However, we found that in mice lacking Fc $\gamma$ receptors I and III, SHe immune serum failed to protect, arguing against direct virus neutralization by SHe-specific antibodies in vivo [22].

It is well known that next to direct neutralization, antibodies can hamper infections by engaging the complement system or Fc $\gamma$ receptors, via their Fc tail, to eliminate viral particles or infected cells. For example, the Fc tail of two monoclonal antibodies that recognize the conserved cysteine-noose of the G protein are essential for in vivo protection [24,25]. The protection by the non-neutralizing 18A2B2 monoclonal antibody was shown to be dependent on the comple- ment system [25]. In contrast, the observation that both Fab fragments and nanobodies ${ }^{\circledR}$ (the antigen binding domain of cameloid heavy chain-only antibodies) that target the Synagis epitope in F inhibit HRSV replication in mice suggests that these antibodies do not depend on their Fc tail for protection [26,27]. By using Fc $\gamma I / I I I^{-/-}$mice we could demonstrate the importance of FcyRI and/or FcyRIII in SHe antibody-mediated control of infection. In mice, alveolar macrophages express both Fc $\gamma$ RI and Fc $\gamma$ RIII whereas NK cells that quickly infiltrate in HRSV-infected lungs only express FcyRIII [28]. Using cell depletion we highlighted an important role for alveolar macrophages, but not NK cells in $\mathrm{SHe}$ immune serum-mediated protection. Intriguingly HRSV might have adopted strategies to escape from both neutralizing and Fc $\gamma \mathrm{R}$-engaging antibodies. HRSV G is also produced as a secreted form ( $\mathrm{sG}$ ). Recombinant viruses that only express membrane-anchored $G$ proteins and not $s G$ were more sensitive to G-specific neutralizing antibodies in vitro but also more sensitive to HRSV or F immune serummediated protection in vivo. This increased sensitivity could be explained by enhanced Fc $\gamma$ receptor-mediated uptake of viral particles or infected cells that are opsonized by anti-G antibodies by leukocytes [29]. It would be of interest to investigate whether $s G$ would also affect $\mathrm{SHe}$ immune serum-mediated protection of HRSV.

In contrast to $\mathrm{F}$ and $\mathrm{G}, \mathrm{SH}$ is hard to detect on the surface of virions [15,22]. This suggests that in contrast to F- and G-specific antibodies, SHe-specific antibodies control HRSV infection by targeting infected cells rather than HRSV virions. Therefore, we propose that SHe-based vaccination reduced HRSV infection by aiding in the clearance of infected cells. In infants, severe and fatal HRSV infections are caused by airway obstruction by plugs containing HRSVinfected cells shed from the epithelium. These lungs lack HRSV-specific $\mathrm{T}$ cells which would normally clear infected cells [3]. Interestingly, airway occlusion by cellular debris also occurs in HRSV-infected New Zealand black mice, which have constitutive deficiencies in macrophage function, and in BALB/c mice with depleted alveolar macrophages [30]. As such SHespecific antibodies that engage $\mathrm{F} c \gamma$ receptor-expressing macrophages to remove HRSV-infected epithelial cells might, in addition to reducing HRSV progeny from these infected cells, also avert airway occlusion.

An important question is whether a vaccine such as $\mathrm{SHe}$, that does not induce neutralizing antibodies but aims at elimination of infected cells, could have a clinical benefit? We and others have shown that nonneutralizing antibodies directed against the ectodomain of the influenza matrix protein 2 (M2e), pro- 
tect against influenza infections by engaging alveolar macrophages to eliminate infected cells via Fc $\gamma$ R I and III [31]. Recently, the therapeutic efficacy of a non-neutralizing human M2e-specific monoclonal antibody (TCN-032) was evaluated in humans by experimental infection. Therapeutic treatment with TCN-032 significantly reduced and shortened viral shedding and symptoms to a similar extent that has been observed for the influenza antivirals; oseltamivir (Tamiflu) and peramivir [32]. These promising results indicate that a vaccine that targets infected cells can have significant clinical benefit upon acute respiratory infections. One can imagine that the possible clinical impact of a therapeutic antibody treatment or a vaccine aiming at controlling viral replication by eliminating infected cells strongly depends on the kinetics of viral replication. In experimentally infected adults, influenza virus shedding peaks at 2 days postinfection, whereas HRSV peaks at 6 days postinfection [33]. In children infected with live attenuated HRSV, viral load peaks at 7-8 days postinfection [34]. This relatively slow kinetics of HRSV infection provides a window of opportunity

\section{References}

1 Nair H, Nokes DJ, Gessner BD et al. Global burden of acute lower respiratory infections due to respiratory syncytial virus in young children: a systematic review and meta-analysis. Lancet 375(9725), 1545-1555 (2010).

2 Liesman RM, Buchholz UJ, Luongo CL et al. RSV-encoded NS2 promotes epithelial cell shedding and distal airway obstruction. J. Clin. Invest. 124(5), 2219-2233 (2014).

3 Welliver TP, Reed JL, Welliver RC Sr. Respiratory syncytial virus and influenza virus infections: observations from tissues of fatal infant cases. Pediatr. Infect. Dis. J. 27(10 Suppl.), S92-S96 (2008).

4 Glezen WP, Taber LH, Frank AL, Kasel JA. Risk of primary infection and reinfection with respiratory syncytial virus. Am. J. Dis. Child. 140(6), 543-546 (1986).

5 Hall CB, Long CE, Schnabel KC. Respiratory syncytial virus infections in previously healthy working adults. Clin. Infect. Dis. 33(6), 792-796 (2001).

6 Falsey AR, Hennessey PA, Formica MA, Cox C, Walsh EE. Respiratory syncytial virus infection in elderly and highrisk adults. N. Engl. J. Med. 352(17), 1749-1759 (2005).

7 Espinoza JA, Bohmwald K, Cespedes PF, Riedel CA, Bueno SM, Kalergis AM. Modulation of host adaptive immunity by hRSV proteins. Virulence 5(7), 740-751 (2014).

8 Wright PF, Gruber WC, Peters M et al. Illness severity, viral shedding, and antibody responses in infants hospitalized with bronchiolitis caused by respiratory syncytial virus. J. Infect. Dis. 185(8), 1011-1018 (2002). to control infection by eliminating infected cells. As SHe specific antibodies display a low binding capacity to HRSV virions, those non-neutralizing antibodies would likely not interfere with HRSV-neutralizing antibodies. Hence, a vaccine that combines both $\mathrm{SHe}$ with either $\mathrm{F}$ or $\mathrm{G}$ might be an intriguing vaccination approach.

\section{Financial \& competing interests disclosure}

B Schepens is a postdoctoral research fellow of FWO-Vlaanderen. Research on HRSV in the authors' laboratory is supported by FWO-Vlaanderen, IWT, IUAP BELVIR project p7/45, Ghent University Special Research Grant BOF12/GOA/014 and IOF F2012/IOF-Advanced/010 grants and by VIB. B Schepens and $X$ Saelens are named as inventors on patent application WO/2012/065997, which relates to use of SHe as a vaccine against respiratory syncytial virus. The authors have no other relevant affiliations or financial involvement with any organization or entity with a financial interest in or financial conflict with the subject matter or materials discussed in the manuscript apart from those disclosed.

No writing assistance was utilized in the production of this manuscript.
9

Hall CB, Walsh EE, Long CE, Schnabel KC. Immunity to and frequency of reinfection with respiratory syncytial virus. J. Infect. Dis. 163(4), 693-698 (1991).

10 Piedra PA, Jewell AM, Cron SG, Atmar RL, Glezen WP. Correlates of immunity to respiratory syncytial virus (RSV) associated-hospitalization: establishment of minimum protective threshold levels of serum neutralizing antibodies. Vaccine 21(24), 3479-3482 (2003).

11 Palivizumab, a humanized respiratory syncytial virus monoclonal antibody, reduces hospitalization from respiratory syncytial virus infection in high-risk infants. The IMpact-RSV Study Group. Pediatrics 102(3 Pt 1), 531-537 (1998).

12 Walsh EE, Hruska J. Monoclonal antibodies to respiratory syncytial virus proteins: identification of the fusion protein. J. Virol. 47(1), 171-177 (1983).

13 Mclellan JS, Chen M, Joyce MG et al. Structure-based design of a fusion glycoprotein vaccine for respiratory syncytial virus. Science 342(6158), 592-598 (2013).

14 Zhang W, Choi Y, Haynes LM et al. Vaccination to induce antibodies blocking the CX3C-CX3CR1 interaction of respiratory syncytial virus $\mathrm{G}$ protein reduces pulmonary inflammation and virus replication in mice. J. Virol. 84(2), 1148-1157 (2010).

15 Rixon HW, Brown G, Aitken J, McDonald T, Graham S, Sugrue RJ. The small hydrophobic $(\mathrm{SH})$ protein accumulates within lipid-raft structures of the golgi complex during respiratory syncytial virus infection. J. Gen. Virol. 85(Pt 5), 1153-1165 (2004).

16 Collins PL, Mottet G. Membrane orientation and oligomerization of the small hydrophobic protein of 
human respiratory syncytial virus. J. Gen. Virol. 74(Pt 7), 1445-1450 (1993).

17 Carter SD, Dent KC, Atkins E et al. Direct visualization of the small hydrophobic protein of human respiratory syncytial virus reveals the structural basis for membrane permeability. FEBS Lett. 584(13), 2786-2790 (2010).

18 Fuentes S, Tran KC, Luthra P, Teng MN, He B. Function of the respiratory syncytial virus small hydrophobic protein. J. Virol. 81(15), 8361-8366 (2007).

19 Triantafilou K, Kar S, Vakakis E, Kotecha S, Triantafilou $\mathrm{M}$. Human respiratory syncytial virus viroporin $\mathrm{SH}$ : a viral recognition pathway used by the host to signal inflammasome activation. Thorax 68(1), 66-75 (2013).

20 Whitehead SS, Bukreyev A, Teng MN et al. Recombinant respiratory syncytial virus bearing a deletion of either the NS2 or SH gene is attenuated in chimpanzees. J. Virol. 73(4), 3438-3442 (1999).

21 Akerlind-Stopner B, Hu A, Mufson MA, Utter G, Norrby E. Antibody responses of children to the C-terminal peptide of the $\mathrm{SH}$ protein of respiratory syncytial virus and the immunological characterization of this protein. J. Med. Virol. 40(2), 112-120 (1993).

22 Schepens B, Sedeyn K, Vande Ginste L et al. Protection and mechanism of action of a novel human respiratory syncytial virus vaccine candidate based on the extracellular domain of small hydrophobic protein. EMBO Mol. Med. 6(11), 1436-1454 (2014).

23 Bukreyev A, Whitehead SS, Murphy BR, Collins PL. Recombinant respiratory syncytial virus from which the entire $\mathrm{SH}$ gene has been deleted grows efficiently in cell culture and exhibits site-specific attenuation in the respiratory tract of the mouse. J. Virol. 71(12), 8973-8982 (1997).

24 Boyoglu-Barnum S, Gaston KA, Todd SO et al. A respiratory syncytial virus (RSV) anti-G protein $\mathrm{F}(\mathrm{ab}$ ')2 monoclonal antibody suppresses mucous production and breathing effort in RSV rA2-line19F-infected BALB/c mice. J. Virol. 87(20), 10955-10967 (2013).

25 Corbeil S, Seguin C, Trudel M. Involvement of the complement system in the protection of mice from challenge with respiratory syncytial virus long strain following passive immunization with monoclonal antibody 18A2B2. Vaccine 14(6), 521-525 (1996).

26 Crowe JE, Jr, Murphy BR, Chanock RM, Williamson RA, Barbas CF, 3rd, Burton DR. Recombinant human respiratory syncytial virus (RSV) monoclonal antibody Fab is effective therapeutically when introduced directly into the lungs of RSVinfected mice. Proc. Natl Acad. Sci. USA 91(4), 1386-1390 (1994).

27 Schepens B, Ibanez LI, De Baets S et al. Nanobodies(R) specific for respiratory syncytial virus fusion protein protect against infection by inhibition of fusion. J. Infect. Dis. 204(11), 1692-1701 (2011).

28 Guilliams M, Bruhns P, Saeys Y, Hammad H, Lambrecht BN. The function of Fcgamma receptors in dendritic cells and macrophages. Nat. Rev. Immunol. 14(2), 94-108 (2014).

29 Bukreyev A, Yang L, Fricke J et al. The secreted form of respiratory syncytial virus $G$ glycoprotein helps the virus evade antibody-mediated restriction of replication by acting as an antigen decoy and through effects on Fc receptor-bearing leukocytes. J. Virol. 82(24), 12191-12204 (2008).

30 Reed JL, Brewah YA, Delaney T et al. Macrophage impairment underlies airway occlusion in primary respiratory syncytial virus bronchiolitis. J. Infect. Dis. 198(12), 1783-1793 (2008).

31 El Bakkouri K, Descamps F, De Filette M et al. Universal vaccine based on ectodomain of matrix protein 2 of influenza A: Fc receptors and alveolar macrophages mediate protection. J. Immunol. 186(2), 1022-1031 (2011).

32 Ramos EL, Mitcham JL, Koller TD et al. Efficacy and safety of treatment with an anti-M2e monoclonal antibody in experimental human influenza. J. Infect. Dis. pii: jiu539 (2014) (Epub ahead of print).

33 Bagga B, Woods CW, Veldman TH et al. Comparing influenza and RSV viral and disease dynamics in experimentally infected adults predicts clinical effectiveness of RSV antivirals. Antiviral Ther. 18(6), 785-791 (2013).

34 Wright PF, Karron RA, Belshe RB et al. Evaluation of a live, cold-passaged, temperature-sensitive, respiratory syncytial virus vaccine candidate in infancy. J. Infect. Dis. 182(5), 1331-1342 (2000). 